\title{
On the predictability of individual events in power law systems
}

\author{
Rumi De*i \\ Material Research Center, Indian Institute of Science, Bangalore-560012, India. \\ E-mail: rumi@mrc.iisc.ernet.in \\ G. Ananthakrishna \\ Material Research Center, Indian Institute of Science, Bangalore-560012, India \\ E-mail: garani@mrc.iisc.ernet.in
}

\begin{abstract}
We consider a modified Burridge-Knopoff model with a view to understand results of acoustic emission (AE) relevant to earthquakes by adding a dissipative term which mimics bursts of acoustic signals. Interestingly, we find a precursor effect in the cumulative energy dissipated which allows identification of a large slip event. Further, the AE activity for several large slip events follows a universal stretched exponential behavior with corrections in terms of time-to-failure. We find that many features of the statistics of AE signals such as their amplitudes, durations and the intervals between successive AE bursts obey power laws consistent with recent experimental results. Large magnitude events have different power law from that of the small ones, the latter being sensitive to the pulling speed.
\end{abstract}

International Conference on Statistical Mechanics of Plasticity and Related Instabilities 31 August - 2 September 2005

Bangalore, India

\footnotetext{
* Speaker.

${ }^{\dagger}$ Present address: Department of Materials and Interfaces, Weizmann Institute of Science, Rehovot 76100 Israel.
} 


\section{Introduction}

Causes of failure of materials and the possibility of predicting them is of interest in science and engineering (electrical breakdown, fracture of laboratory samples to engineering structures, etc). This is particularly important in seismology due to the enormous damage earthquakes can cause. Indeed, predicting earthquakes has been of interest to geophysics for a long time. At a practical level, this amounts to identifying useful precursors at a statistically signifi cant level. The absence of useful precursors could possibly arise due to the inherent limitations set by measurement processes [1]. Even so, there has been records of individual earthquakes where precursor effects have been reported [1]. Some insight into the dynamics of earthquakes has been possible by mapping the problem to fracture processes. In the case of fracture, the nucleation and propagation of cracks culminates in the failure of the material. In such situations, the nondestructive nature of acoustic emission (AE) is a very convenient tool for studying the process as the emitted signals are sensitive to the microstructural changes taking place inside the system [2, 3]. Such studies have shown that it is possible to follow the nucleation and growth of fracture by imaging the fracture process (through an inversion process of arrival times) [П]. In addition, the statistics of the AE signals exhibit a power law [2, 4, 5] similar to the Gutenberg-Richters law for the magnitudes of earthquakes. One aim of these studies has been to look for precursor effects [1] 6]. Thus, most laboratory studies on $\mathrm{AE}$ relevant to earthquake dynamics are on rock samples subjected slip with appropriate geometry [3].

Apart from the power laws observed in AE signals during fracture, acoustic activity of unusually large number of situations exhibit power laws in systems as varied as volcanic activity [7], microfracturing process [8, 9], collective dislocation motion [10] and martensitic transformation [11] to name a few. Though the general mechanism attributed to $\mathrm{AE}$ is the release of stored strain energy, the details are system specifi c. Thus, the ubiquity of the power law statistics of AE signals suggests that the details of the underlying processes are irrelevant. One framework which unifi es such varied situations is that of the self-organized criticality (SOC) [17]. This approach has been successful in explaining the statistical self similarity of the seismic process reflected in the Gutenberg-Richter's law for earthquake magnitudes [13], as also the power laws in other systems 17, 8, 9, 10, 11, 14]. However, given that earth is in a SOC state, at a conceptual level, doubts have been raised about the possibility of predicting an earthquake. A recent debate on this subject concluded that deterministic prediction of individual earthquakes is unrealistic [15]. Clearly, the lack of predictability is applicable to all power law systems. However, in the general context of failure, time-to-failure models have been used for the prediction of failure [16]. These models are thought to be applicable to earthquake like situations also. There are some theoretical efforts to look for precursor effects before the onset of large avalanches in SOC type models as well [17, 18].

The power law statistics of the AE signals (on rock samples) is believed to result from slip events. However, there are no phenomenological models that mimic AE bursts. One other interesting feature of earthquake magnitudes is the change in the power law exponent for small and large magnitudes [19, 20]. Laboratory studies on AE signals on rock samples also appear to indicate such a change [21]. Finally, recent studies on AE also show that the exponent value is sensitive to the deformation rate [22]. To the best of our knowledge, there has been no explanation of these observations. Here, we introduce an additional dissipative term into the Burridge-Knopoff (BK) 

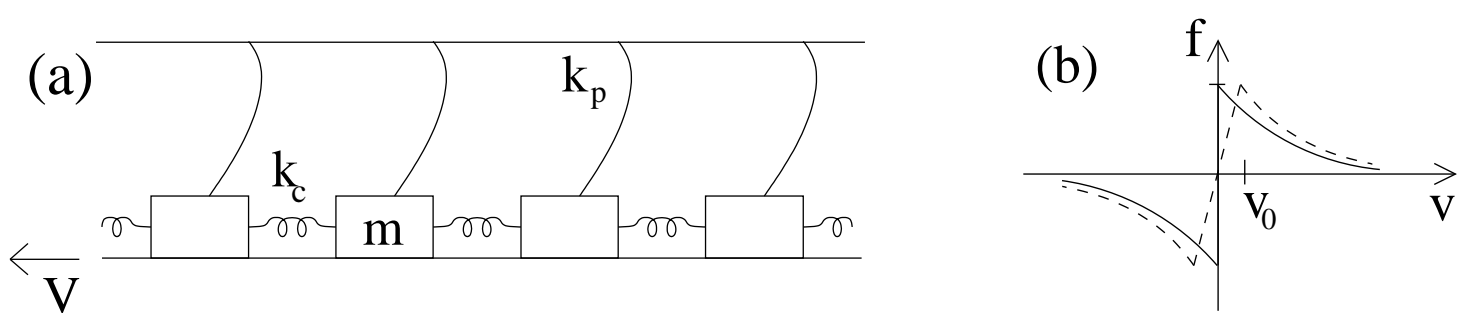

Figure 1: (a) The Burridge-Knopoff spring block model. (b)Friction laws: The coulomb friction law at zero velocity beyond which a smooth velocity weakening friction law (solid line). Dashed curve describes a creep branch with a similar velocity-weakening behavior.

model which captures the main features of AE signals. This also helps us to identify a possible precursory effect.

It is known that slip events result due to deformation and /or breaking of asperities resulting in an accelerated motion of the local areas of slip. Since AE is due to the release of the built-in strain energy as the system surmounts the threshold, we consider this accelerated motion during a local slip as responsible for acoustic emission. Such a rapid movement prevents the system from attaining a quasi-static equilibrium. This also implies that there are dissipative forces that resist this abrupt motion. We introduce the Rayleigh dissipation functional which depends on the gradient of the local strain rate [23] to account for the dissipation arising from the rapidly moving blocks in the BK model for earthquakes [24, 25]. Indeed, such a dissipative term has been successful in explaining the power law statistics of the AE signals during martensitic transformation [14].

\section{Model}

The Burridge-Knopoff model for earthquakes [24] and its variants have been studied in detail by many authors [25]. It consists of a chain of blocks of mass $m$ coupled to each other by coil springs of strength $k_{c}$ and attached to a fi xed surface by leaf springs of strength $k_{p}$ (Fig. 1a). The blocks are in contact with a rough surface moving at constant speed $V$. The velocity-dependent friction force ' $f$ ' operates between the blocks and the surface. We use two forms of frictional force schematically shown by the solid and dashed curves shown in Fig. 1 .

Here, we introduce an dissipation associated with the rapid slip events represented by the Rayleigh dissipative functional [23] $R=\frac{\gamma_{c}}{2} \int\left(\frac{\partial \dot{u}(x)}{\partial x}\right)^{2} d x$. Then, in the notation of Ref [25], the equations of motion can be written as

$$
\ddot{U}_{j}=l^{2}\left(U_{j+1}-2 U_{j}+U_{j-1}\right)-U_{j}-\phi\left(2 \alpha v+2 \alpha \dot{U}_{j}\right)+\gamma_{c}\left(\dot{U}_{j+1}-2 \dot{U}_{j}+\dot{U}_{j-1}\right),
$$

where $U_{j}$ is the dimensionless displacement of the $j^{\text {th }}$ block, $v$ is the dimensionless pulling velocity, the ratio of the slipping time to the loading time, $l^{2}=k_{c} / k_{p}$ represent the stiffness ratio and the parameter describing the rate of velocity-weakening in the friction is $\alpha$, The last term arises from $R(t)$, the additional dissipative term introduced to mimic the AE bursts and $\gamma_{c}$ is the scaled dissipation coeffi cient. ( The over dot refers to differentiation with respect to dimensionless time variable.) 
This model without the last term has been extensively studied [24, 25]. Starting from random initial conditions, slip events ranging from one-block event to those extending over the entire fault ('large events', occurring roughly over one loading period of $\tau_{L} \sim 2 / v$ ) are seen in the steady state. These earthquake-like events mimic the empirical Gutenberg-Richter law.

\section{Results}

Simulations have been carried out using fourth-order Runge-Kutta method with open boundary condition. Random initial conditions are imposed. After discarding the initial transients, long data sets are recorded when the system has reached a stationary state. The parameters used here are $l=10, \alpha=2.5, N=100$ for two sets of values of $v=0.01$ and 0.001 for range of values of $\gamma_{c}$. The calculations have been carried out for both the frictional laws shown in Fig. 1b. In the creep case, the creep branch ends at a value of $v \sim 10^{-7}$ beyond which the velocity weakening law operates. The modifi ed BK model produces the same statistics of slip events as that without this term as long as the value of $\gamma_{c}$ is small, typically $\gamma_{c}<0.5$. The results presented here are for $\gamma_{c}=0.02$.
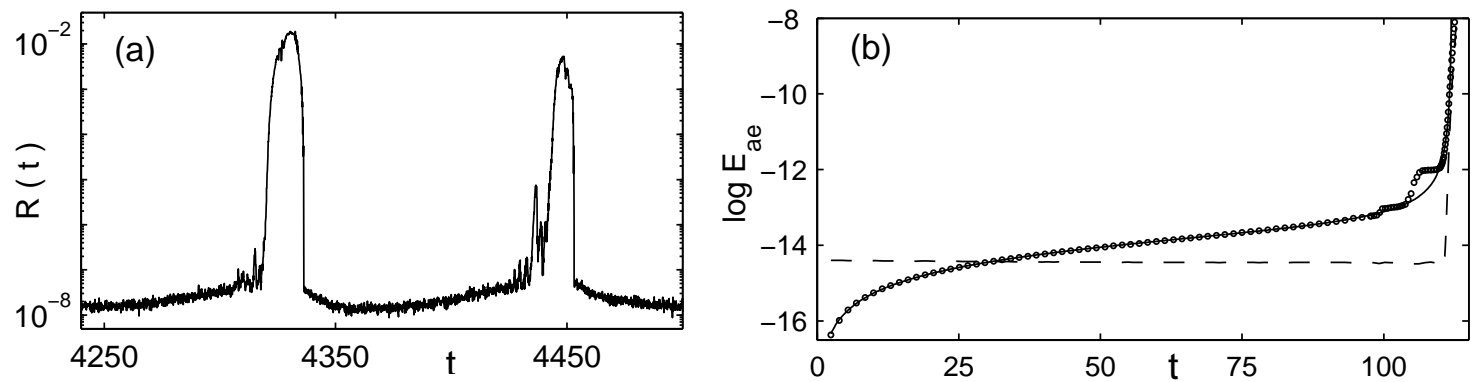

Figure 2: (a) R(t) as a function of t. (b) $\log E_{a e}$ as a function of time t (o) along with the fit (solid line) for the friction law with creep branch. Dashed line corresponds to the mean kinetic energy.

Since the rate of energy dissipated [23] due to local accelerating blocks is given by $d E_{a e} / d t=$ $-2 R(t)$, we calculate $R(t)=\left(\gamma_{c} / 2\right) \sum_{j}\left(\dot{U}_{j}-\dot{U}_{j-1}\right)^{2}$. We fi nd that the energy dissipated occurs in bursts which is similar to the acoustic emission signals. A plot of $R(t)$ is shown in the Fig. 2a for the case when the frictional law has a creep branch. A gradual increase in the activity of the energy dissipated can be seen to accelerate just prior to the occurrence of a 'large' slip event. This feature is seen for all slip events of observable magnitude which we refer as large. This suggests that $R(t)$ can be used as a precursor for the onset of a slip event observed in experiments on rock samples [6]. As the energy dissipated is noisy, a better quantity for the analysis is the cumulative energy dissipated $E_{a e}(t)\left(\propto \int_{0}^{t} R\left(t^{\prime}\right) d t^{\prime}\right)$. This grows in a stepped manner and as we approach a slip event, $E_{a e}$, increases rapidly with the steps becoming increasingly visible. A plot of $\log E_{a e}$ is shown in Fig. $2 b$ along with a fi $t$ having the functional form (continuous line),

$$
\log E_{a e}(t)=-a_{1} t^{-\alpha_{1}}\left[1-a_{2}\left|\left(t-t_{c}\right) / t_{c}\right|^{-\alpha_{2}}\right] .
$$

Here, the crucial parameter $t_{c}$ is the time of occurrence of the event and $t$ is time measured from some initial point after a slip event. The constants $a_{1}, a_{2}, \alpha_{1}$ and $\alpha_{2}$ are determined by a fit to the data. It is clear that the fit is striking. Given a reasonable stretch of the data, the initial increasing 


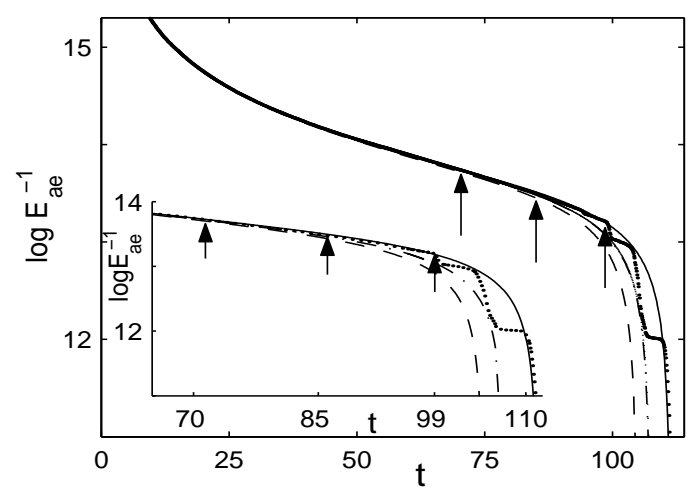

Figure 3: A plot of $\log E_{a e}^{-1}$ versus $t$. Inset shows the enlarged section at time $t_{1}(-), t_{2}(-\cdot)$ and $t_{3}$ (solid line). Data shown $(\cdot)$ is indistinguishable from the fit till $t_{3}$.

trend in $\log E_{a e}$ is easily fi tted to a stretched exponential, i.e., $-a_{1} t^{-\alpha_{1}}$. The second term is introduced to account for the observed rapid increase in the activity as we approach the time of failure. As the mean kinetic energy is a good indicator of the failure time, we have shown this by a dashed line. It is clear that the estimated $t_{c}$ agrees quite well with that of the mean kinetic energy.

A proper estimate of the warning time requires that the values of the four constants $a_{1}, a_{2}, \alpha_{1}$ and $\alpha_{2}$ do not change in time signifi cantly. Indeed, we fi nd that these constants change very little given a data $E_{a e}(t)$ over a reasonable initial stretch of time. Only $t_{c}$ changes. Still, we are left with the problem of obtaining a best estimate of $t_{c}$. This is done as follows. Consider the plot of $E_{a e}^{-1}(t)$ shown in Fig. 3. Given the data till $t=t_{1}$ ( the first arrow shown in Fig. \$), we fi nd that the four parameters are already well determined (within a small error bar). A fit to Eq. (2) also gives $t_{c}^{(1)}$ at $t_{1}$. One such curve is shown by a dashed line with the arrow shown at $t_{1}$. The value of $t_{c}^{(1)}$ is only an estimate based on the data till $t_{1}$. However, as time progresses, the data accumulated later usually deviates from the predicted curve beyond $t_{1}$ if $t_{c}^{(1)}$ is inaccurate. This is case for the fi t till $t_{1}$ and $t_{2}$ for instance shown in Fig. 3. If on the other hand, the deviation of the predicted curve from the accumulated data decreases with passage of time within the error bar, then, the value of $t_{c}$ is likely to be accurate. Indeed, the extrapolated (continuous) curve shown in Fig. 3 corresponding to data fit till $t=\hbar$ with the corresponding predicted $t_{c}^{(3)}$ is seen to follow the data very well. (Usually, this is followed by a sudden decrease in $E_{a e}^{-1}$ which is again an indication of a possible large event, but the general trend soon follows the extrapolated curve.) Then, one can take $t_{3}$ to be the warning time of the impending large event. From the data shown in Fig. 3 , the actual $t_{c}$ is 110.4 where as the predicted $t_{c}$ is 111.6. Thus, the percentage error in the prediction of the onset of the a large event $\sim 1 \%$. The power law nature of $E_{a e}$ also suggests that the approach to different events is universal. We fi nd that the data corresponding to different events collapses into a single curve given by $a_{1}^{-1} \log \left[E_{a e}(0) / E_{a e}(\tau)\right]=\tau^{-\alpha_{1}}\left[1-a_{2} \mid(\tau-1)^{-\alpha_{2}}\right]+a_{1}^{-1} \log E_{a e}(0)$ in terms of $\tau=t / t_{c}$. This is shown in Fig. $\bigoplus$ along with the fit for three different events.

For the Coulomb friction law, we fi nd that $R(t)$ is much more noisy. A plot of $\log E_{a e}$ for one large event is shown in Fig. 5. Following the same procedure, we fi nd that we can fi x only $q$ and $\alpha_{1}$ reasonably well given an initial stretch of data. However, the parameters $a_{2}$ and $\alpha_{2}$ also converge within some error bar which is more than that for the creep case. Although, the changes 


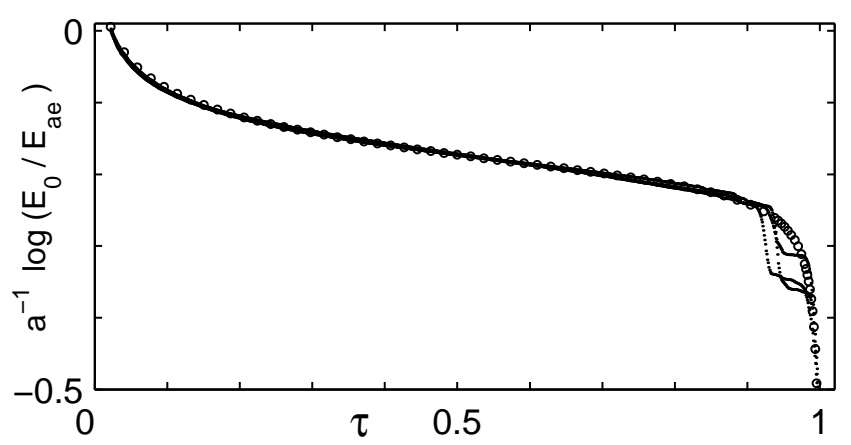

Figure 4: Collapsed data using $a_{1}^{-1} \log (E(0) / E(\tau))$ versus $\tau$ for three different events along with the fit shown by (o) for friction law with creep.

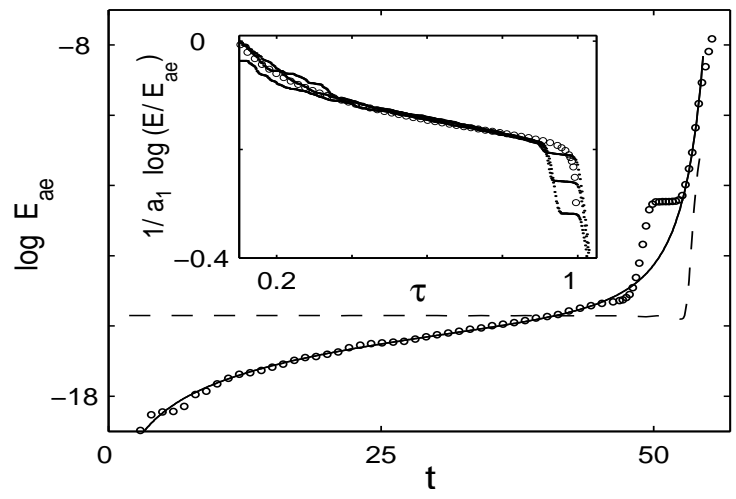

Figure 5: $\log E_{a e}$ as a function of time $\mathrm{t}(\mathrm{o})$ along with a fit (solid line) for the coulomb friction . Dashed line indicates the mean kinetic energy. The inset shows the collapsed curve for three different events along with a fit (o).

in $t_{c}$ with $t$ is more than the previous case, the $t_{c}$ is fi xed the same way, but the error is larger than the previous case. The data along with the fi $\mathrm{t}$ (up to the point shown by arrow) is clearly seen to mimic the rapid increase in $\log E_{a e}$. The data collapse for several such curves (for $a_{1}^{-1} \log \left[E_{a e}(0) / E_{a e}(\tau)\right]$ ) is also shown in the inset along with the fi t. (The scatter in the collapsed data is reasonable except for the initial part of the data.) However, we do fi nd that this procedure does pose problems in a few specifi c set of events for which approach to the large event is very noisy.

We now consider the statistics of the energy bursts. The distribution of the magnitudes of $R(t)$ shows a power law $D(A) \sim A^{-m}$, where $D(A)$ is the number of events between $A$ and $A+d A$. Instead of a single power law anticipated, we find that the distribution shows two regions, one for relatively smaller amplitudes and another for large values shown by two distinct plots in Fig. 6a. ( This is for the Coulomb frictional law for $v=0.01$. Similar results are obtained when the frictional law has a creep branch.) The value of $m$ for the small amplitudes region $\left(<10^{-4}\right)$ is $\sim 1.78$, signifi cantly smaller than that of large amplitudes which is $\sim 2.09$. This qualitative feature is consistent with the recent experimental results on rock samples [21]. Indeed, this is similar to the well known observation in the case of seismic moments where a deviation from the power law 

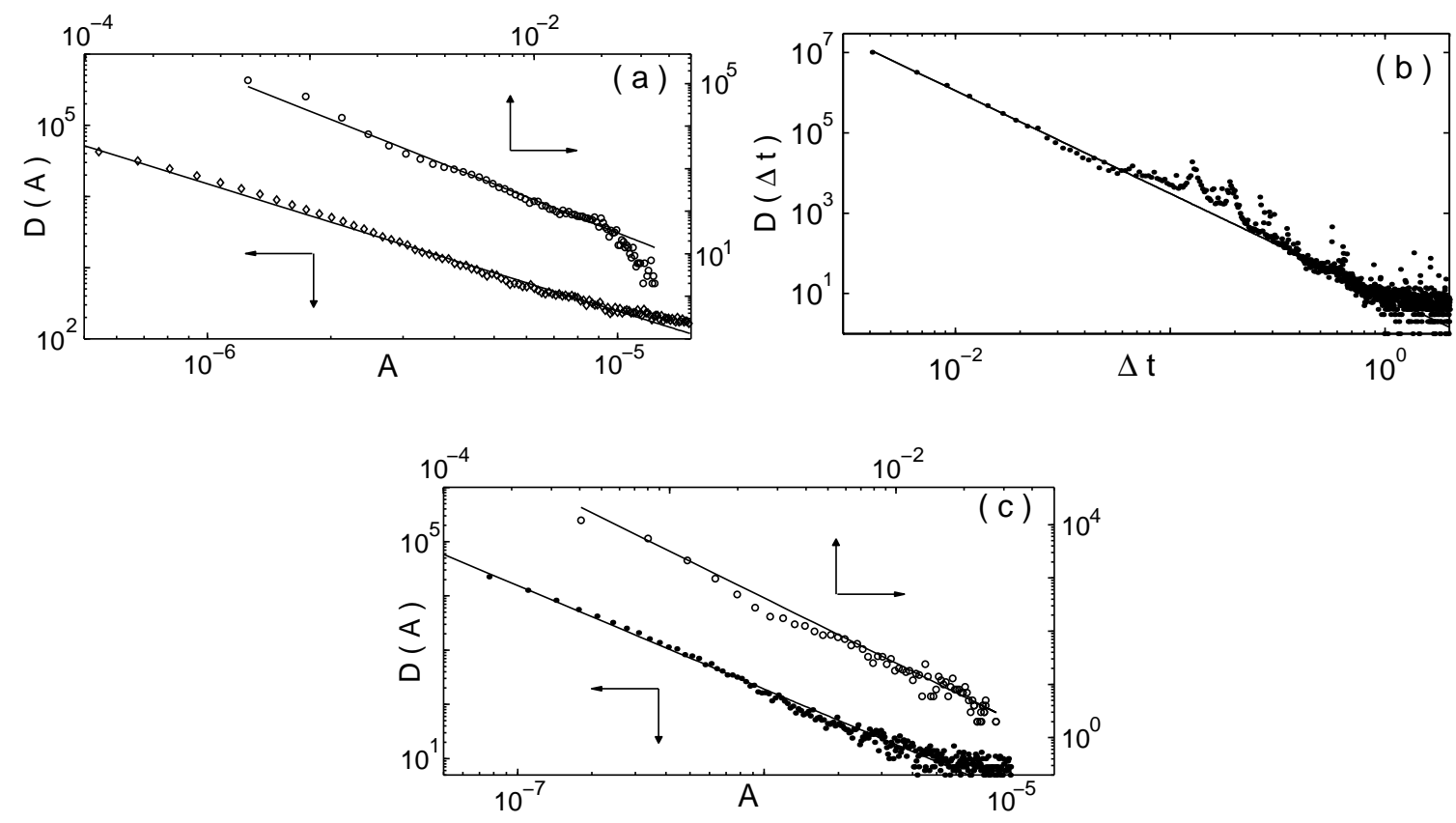

Figure 6: (a)Distribution of amplitude of $R(A), D(A)$ versus A. $(\diamond)$ indicates small amplitudes and (o) shows large amplitudes for $v=0.01$, (b) Distribution of time intervals of AE events and (c) $D(A)$ versus A for $v=0.001,(\bullet)$ corresponds to the small amplitudes and (o) that for large.

for lower magnitudes beyond a certain value ( $>7.0$ on the Richter scale [19, 20]) is noted. Finally, one other quantity of interest is the time interval between the events which we have calculated. This is shown in Fig. $6 \mathrm{~b}$ for $v=0.01$. There appears to single power law with and exponent 2.6. However, there is considerable scatter in the mid region corresponding crossover in the power laws from small to large amplitude region. For smaller pulling speeds, we see two different scaling regimes as for the amplitude.

Recently Yabe et. al. [22] have noted that the exponent value in the small amplitude region increases with decreasing deformation rate in contrast to the large amplitude region. In order to check this, we have performed run for $v=0.001$ for which the data (for the Coulomb friction law ) is shown in Fig. 6c. While the exponent for small amplitude regimes is 1.91 higher than that for $v=0.01$, the exponent for larger amplitudes is insensitive. This result can be physically explained by analyzing the influence of the pulling velocity on slip events of varying sizes. We fi rst note that Rayleigh dissipation function is the gradient of local slipping rates. From the arguments presented in Ref. [25], one knows that the velocity of one block event is proportional to $v$. Further, as the neighboring blocks are at rest, the number of such events are fewer in proportion to the pulling speed, both of which are evident from Fig. 6a and Fig. 6c. When we consider the two block events, it clear that the difference in the velocities of the two blocks being of similar magnitude contributes very little and only edges contribute. In a similar way, it can be argued that for slip events of fi nite size, the extent of the contribution to $R(t)$ is decided by the ruggedness of the velocity profi le within the slipping region; it is lower if the velocity is smoother. The ruggedness of the velocity profi le, however, is itself decided by how much time the system gets to 'relax'. At lower pulling 
velocities, there is suffi cient time for the blocks to relax compared to higher pulling velocities. Thus, the velocity profi le within slip event of certain magnitude tends to be much more smooth at low $v$ compared to at higher higher $v$ as in the latter case it does not allow for full relaxation (to attain near quasi-stationary state).

\section{Discussions}

In summary, within the scope of this model, we have shown that acoustic emission could be used as a possible precursor for detecting an event during the process of failure. In the case where the friction includes a creep branch, we fi nd that the time of failure can be predicted quite accurately. In the coulomb case, the energy dissipated is quite noisy. Even then, the predicted failure time is quite reasonable. At the fi rst sight, the predictability aspect appears to be surprising considering the fact the statistics of the events exhibits a power law. However, the data collapse for different events clearly suggests that the dynamics of approach to large events is itself universal and scale invariant. This scale invariant form implies that all events of detectable magnitude is describable by the same equation, the only limitation being the ability to detect. One limitation of Eq. (2) is that the magnitude of the energy dissipated appears to bear no correlation with the magnitude of the slip event as larger events often show higher $R(t)$. For instance, even when the ratio of the kinetic energies between two events is four orders smaller, the energy dissipated $R(t)$ in the two cases do not scale in proportion. For the same reason, the model is unable to predict the magnitude of the event. We stress that this precursor effect is absent in the total kinetic energy or other variables. We also expect these results to be applicable at the laboratory level in failure of materials and structures. It is worth noting that the form of approach to failure is different from that given by Huang et al [16]. Apart from this, the model also predicts that there are two exponent values one for small amplitudes and another for large amplitudes consistent with experimental results [21]. We also fi nd that the exponent value corresponding to low amplitudes to be much more sensitive to the pulling speed than that at large amplitudes. This dependence on the pulling speed has been be traced to the form of $R(t)$, namely, the gradient of the local strain rate. Finally, our analysis shows that the exponent values of the bursts of acoustic energy is not the same as that of the event size distribution (as used in Ref.[25]).

\section{Acknowledgment}

The authors wish to acknowledge the support from Department of Science and Technology (DST). GA wishes to acknowledge the grant of Raja Ramanna Fellowship.

\section{References}

[1] I. Main, Statistical physics, seismogenesis and seismic hazard, Rev. Geophys. 34 (1996) 433.

[2] K. Mogi, Study of the elastic shocks caused by the fracture of heterogeneous materials and its relation to earthquake phenomena, Bull. Earthquake Res. Inst. 40 (1962) 125.

[3] C. H. Scholz, Microfracturing and the inelastic deformation of rock in compression, J. Geophys. Res. 73 (1968) 1417. 
[4] A. Lockner, J. D. Byerlee, V. Kuksenko, A. Ponomarev, and A. Sidorin, Quasi-static fault growth and shear fracture energy in granite, Nature 350 (1991) 39; A.Lockner, The role of acoustic emission in the study of rock fracture, Int. J. Rock Mech. Min. Sci. and Geomech. Abstr. 30 (1993) 883.

[5] C. H. Scholz, The frequency-magnitude relation of microfracturing in rock and its relation to earthquakes, Bull. Seismol. Soc. Am. 58 (1968) 399.

[6] P. R. Sammonds, P. G. Meredith, and I. G. Main, Role of pore fluids in the generation of seismic precursors to shear fracture, Nature 359 (1992) 228.

[7] P. Diodati, F. Marchesoni, and S. Piazza , Acoustic emission from volcanic rocks: an example of self-organized criticality, Phys. Rev. Lett. 67 (1991) 2239.

[8] A. Petri, G.Paparo, A. Vespignani, A. Alippi, and M. Constantini, Experimental evidence for critical dynamics in microfracturing processes, Phys. Rev. Lett. 73 (1994) 3423.

[9] A. Garcimartin, A. Guarino, L. Bellon, and S. Ciliberto, Statistical properties of fracture precursors, Phys. Rev. Lett. 79 (1997) 3202.

[10] M.-Carmen Miguel, A. Vespignani, S. Zapperi, J. Weiss, J. R. Grasso, Intermittent dislocation flow in viscoelastic deformation, Nature 410 (2001) 667.

[11] E. Vives, J. Oktin, L. Manosa, I. Rafols, R. Perez-Magrane, and A. Planes , Distributions of avalanches in martensitic transformations, Phys. Rev. Lett. 72 (1994) 1694.

[12] P. Bak, C. Tang, and K. Wiesenfeld, Self-organized criticality: an explanation of 1/f noise, Phys. Rev. Lett. 59 (1987) 381.

[13] P. Bak and C. Tang, Earthquakes as a self-organized critical phenomenon, J. Geophys. Res. 94 (1989) 15635 .

[14] R. Ahluwalia and G. Ananthakrishna, Power-law statistics for avalanches in a martensitic transformation, Phys. Rev. Lett. 86 (2001) 4076.

[15] Nature debate, Is the reliable prediction of individual earthquakes a realistic scientific goal? http://helix.nature.com/debates/earthquake (1999).

[16] Y. Huang, H. Saleur, C. Sammis, and D. Sornette, Precursors, aftershocks, criticality and self-organised criticality, Europhys. Lett. 41 (1998) 43.

[17] J. Rosendahl,M. Vekic, and J. E. Rutledge, Predictability of large avalanches on a sandpile, Phys. Rev. Lett. 73 (1994) 537.

[18] M. Acharyya and B. K. Chakrabarti, Growth of breakdown susceptibility in random composites and the stick-slip model of earthquakes: Prediction of dielectric breakdown and other catastrophes, Phys. Rev. E 53 (1996) 140.

[19] J. F. Pacheco, C. H. Scholz, and L. R. Sykes, Changes in frequency-size relationship from small to large earthquakes, Nature 355 (1992) 71.

[20] A. Sornette and D. Sornette, Self-organized criticality and earthquakes, Europhys. Lett. 9 (1989) 197.

[21] Y. Yabe, N. Kato, K. Yamamoto, and T. Hirasawa, Effect of sliding rate on the activity of AE during stable sliding, Pure Appl. Geophys. 160 (2003) 1163.

[22] Y. Yabe, Rate dependence of AE activity during frictional sliding, Geophys. Res. Lett. 29 (2002) No.10.1029/2002GL014865.

[23] Landau L. D. and Lifschitz E. M., Theory of Elasticity, Pergamon, Oxford, 1986. 
[24] R. Burridge and L. Knopoff, Model and theoretical seismicity, Bull. Seismol. Soc. Am. 57 (1967) 341.

[25] J. M. Carlson, and J. S. Langer, Properties of earthquakes generated by fault dynamics, Phys. Rev. Lett. 62 (1989) 2632; B. E. Shaw, J. M. Carlson, and J. S. Langer, Patterns of seismic activity preceding large earthquakes, J. Geophys. Res. 97 (1992) 479. 